\title{
A Review: Targeted Cancer Therapy as a Fight Against Brain Tumor
}

\author{
Rehana Hanif ${ }^{1}$, Yamna Saud Anwari ${ }^{1}$, Qurat-ul-ain Iqbal ${ }^{1}$, Rabia kalsoom ${ }^{1}$, Rana Khalid Iqbal $^{1}$ \\ and Farhana Naureen Anwar ${ }^{2}$ \\ ${ }^{1}$ Institute of Molecular Biology \& Biotechnology Bahauddin Zakariya University, Pakistan
}

${ }^{2}$ Department of Pharmacy Bahauddin Zakariya University, Pakistan

*Corresponding author: Rana Khalid Iqbal, Institute of Molecular Biology \& Biotechnology Bahauddin Zakariya University, MultanPakistan.

To Cite This Article: Rana Khalid Iqbal. A Review: Targeted Cancer Therapy as a Fight Against Brain Tumor. Am J Biomed Sci \& Res. 2019 - 3(3). AJBSR.MS.ID.000672. DOI: 10.34297/AJBSR.2019.03.000672

Received: April 13, 2019 | Published: June 11, 2019

\begin{abstract}
The brain tumor is the second most deadly disease for causing death among cancer patients. It can be treated by conventional treatment techniques i.e. chemotherapy, neurosurgery, and radiotherapy but with some improvement, it mostly results in the increase of medical problems by non-specific targeting of normal cells in addition to the cancerous cells. Different CNS diseases include cerebrovascular disease, Parkinson's disease, epilepsy, Alzheimer disease, and other neurogenerative disorders. Despite advance ongoing research, a number of patients are dying every year by these diseases. Different techniques are used including invasive and non- invasive to treat these diseases. Molecular targeting agents are being used which have the advantages of easily crossing multiple barriers i.e. Brain blood barrier (BBB), cerebrospinal fluid (CSF) and brain bloodtumor barrier to reach the malignant site, low toxicity and better efficacy than conventional treatment techniques. Antibodies, nanoparticles and proton therapy can be used for specific targeting of tumor antigens which spare normal cells from the diseased ones. Although there are some issues i.e. difficulty in crossing multiple barriers, toxicity profiles, sometimes non-specific delivery, degradation of therapeutic agents by the enzymes. Research work is in progress to solve these issues.
\end{abstract}

Keywords: Brain blood barrier; Brain tumor; Nanoparticles; Glioblastoma

\section{Introduction}

The Brain tumor can be treated by surgery, radiation, and chemotherapy. With the implications of all these strategies, the overall survival rate of a glioblastoma patient is only 14.6 months after diagnosis [1]. Moreover, the brains of children are very sensitive to tolerate these intense treatment strategies. So, there is a need to discover such agents that particularly target the malignant cells while causing no harm to normal cells [2]. Treatment of brain tumor is very difficult due to certain barriers that make it difficult for the therapeutic agent to reach the targeted infectious cell or tissue.

The major obstacle is the Brain Blood Barrier (BBB) that does not allow any drug or foreign substance to reach to the brain [3]. Another barrier is the enzymes present in BBB e.g. phosphatase enzyme that attack these molecules due to these barriers. So, most drugs have no access to the CNS [4]. Now the particles having to target specificity, i.e. antibody/Nanoparticles can cross this barrier and can reach these affected cells [5]. But the passage of targeted drugs. i.e. protein, oligonucleotides, peptides is interrupted very often [6].

With the identification of tumor-specific antigens, it became possible to develop such therapeutic methods that selectively target diseased cells. Targeted therapies are being used for the treatment of the brain tumor that specifically targets the pathways that are responsible for the disease [7].

\section{Brain Tumor}

Brain tumor includes diminishing distinction and deviant multiplication of precursor cells. Recent research has described that cancer-causing events make the change from asymmetrical to symmetrical cell division. The most common malignant tumors in adults and children are Glioma and Medulloblastoma (MB). Glioma is further characterized to Grade I, II, III and grade IV [8]. Grade I 
is called Pilocytic astrocytoma and mostly occur in children. On the other hand, Grade II and III termed as oligodendroglia astrocytoma, oligoasrocytoma ependymoma is reported to occur in both adults and children's equally [5] same as vast depict of MBs has accepted numerous subgroups of tumors [9]. Inspire of Glioma and MB cells exhibit definite glial and neuronal makeup, both types of brain tumors have been delineated to emerge from neural indication, competent to show neurogenesis. The ventricle present between the forebrain and spinal canal form glial cells [10]. Glial Fibrillary Acid Protein (GFAP)showing neural stem cells (NSCs) that are also termed as Type B1 cells are considered to sustain asymmetrical cell division to produce transit magnifying ancestors (TAPS, Type C cells), that stimulate differentiation into unripe neuroblast [11].

\section{Obstacles in brain tumor treatment}

There are many obstacles to the treatment of brain tumor due to the complex nature of the brain, different morphological characteristics of each type of brain tumor, inaccessibility of therapeutic agents to the tumor cells, difficulty in specifically targeting of tumor cells while giving no harm to surrounding normal cells and sometimes due to the development of resistance against chemotherapeutics agents [12]. One of the major obstacles is the brain blood barrier.

\section{Brain blood barrier}

Brain blood barrier (BBB) which protects the brain can be considered as the major obstacle to the therapeutic agent [3]. It includes endothelial cells, pericytes, astrocytes, and neurons. Brain capillary endothelial cells (BCEC) are the major boundary between the brain and cellular tissues. The function of pericytes is to maintain and support the BCEC [13]. Astrocytes are the nonneuronal cells that release signal to regulate the permeability of endothelium [1]. Neurons supply capillary to the BBB with nerves. So, the major barrier component is BCEC [14]. Another barrier is the enzymes present in the brain blood e.g. phosphatase enzyme that attack foreign molecules and limit their access to the CNS [6]. With the formation of brain tumor, infectious cells begin to attack healthy brain tissue and after growing to a certain volume they also give harm to the BBB and formation of blood-brain tumor barrier occurs [15]. Another barrier that limits the entry of therapeutic agents is the blood-cerebrospinal fluid barriers (CSF) [16]. It is also composed of epithelial cells which form a barrier and do not allow any therapeutic agent to enter the brain parenchyma. So, most of the molecules failed to reach the targeted site [17].

\section{Targeted strategies for the Treatment of Brain Tumor}

The mediate general survival of patients with glioblastoma (GBM) is only 14.6 months after recent multimodal treatment aggressive surgical abscission followed by coincident or consecutive radiation temozolomide chemotherapy [18].

\section{BBB targeting strategies}

The Blood Brain Barrier is not only responsible for the protection of the brain from harmful substances but is also involved in providing the necessary nutrients to the brain. It is the main challenge in drug delivery to the brain [15].

\section{Absorptive-Mediated transcytosis}

It furnishes a way for the transport of drugs beyond BBB by cationic protein or cell-Penetrating peptide. Classic cationic bovine serum albumin accumulated with pegylated nanoparticle (CBSANP) was designed for brain targeting drug delivery [19]. It was stated that plasmid PORF-hTRAIL (pDNA) subsume (CBSA-NP) abreast with glycoprotein in brain and tumor micro variability and assembled in tumor cells $[19,20]$ arrogate other cationic protein called wheat germ agglutinin (WGA) associated to the surface of liposomes and also reveal elevated BBB transporter.CPP has been used to lessen the lipophilic barrier of the cell membrane and transport large diversity of baggage which involves peptides, DNA, antibodies, toxins and Nano drug carrier such as liposomes and micelles [21].

\section{Transporter-mediated transcytosis}

It is substrate selective so the drugs that intimate the endogenous substances would be delivered to the brain. Glucose Transporters (GLUT) which ease the delivery of glucose from the blood to the brain, have inclusive approach use in brain quarry. Another chief system is choline transporter which ties up positively charged ammonium group or simple cations [22]. The glazed nanoparticle was skill full to hybrid an in vitro replica of BBB (Bovine BCEC).

\section{Receptor-mediated transcytosis}

This is the technique that involves the only drug delivery that bosom imitates the endogenous carrier and then delivered to the brain. One of the most accepted receptor-mediated transcytoses for brain targeting is the Transferrin Receptor (TfR) [23]. These are stacked with polyphosphoester Hybrid Micelles (TPM). It expresses a strong anti- Glioma activity [11]. The low-density lipoprotein (LDC) and Receptor-related protein (LRP) have been noticed to mediate delivery of different ligand associated with Nano carrier beyond the BBB [24].

\section{BBTB targeting strategies and drug delivery}

It is present between brain tumor tissues and micro vessels devised by extremely peculiar endothelium cells (ECs) restricting the transforming of the hydrophilic molecule to tumors [25]. The techniques suggested for BBTB are mostly constructed on receptor highly intimate on the tumor as epidermal growth factor receptor and integrity. Arginine glycine aspartic acid (RGD) is going to be used for targeted drug transformation to the brain.

\section{Antibody-Targeted Therapeutics}

Antibodies can be used for this purpose, which can easily identify the foreign toxic substance. It can easily cross multiple barriers including BBB and selectively accumulate in tumor cells [26]. As in the brain tumor, new blood vessels are also formed termed as angiogenesis assisting in growth and proliferation of brain tumor. These molecular targets inhibit angiogenesis as a treatment of the brain tumor. An antibody named as Bevacizumab VEGF (Vascular endothelial growth factor) is very efficient because it targets the mediator of angiogenesis [1]. Cetuximab IgG1 antibody can block 
this receptor [1]. EPGR (Epidermal growth factor receptor) show interaction with the protein kinase $\mathrm{C}$ which signals to activate transcription. Panitimumab and Erlotinib can also be used as an inhibitor of EPGR. Erlotinib can be used in combination with radiotherapy to treat glioma and be tolerated by the patients. It can be taken orally. Nimotuzumba can also especially target tumor cells due to the overexpression of EPGR and give no harm to cancer cells [2].

\section{Nanoparticle targeted therapy}

Nano Technology put great work on brain Tumor and Open the doors for future treatments and researches for the brain tumor. Nano Particles especially play a distinct role in Brain tumor's treatment, surgery and pharmacology researches. Today researches in Nanoparticles have proved a major contributor role for more future work on the brain tumor.

\section{Importance of Nano Particles}

Nanoparticles are important in the treatment of the brain tumor because they can be used by two mechanisms:

a. Specific mechanisms

b. Nonspecific mechanisms

Specific mechanism based on the interaction of antigens on the surface of nanoparticles with tumor cell receptors. Non-specific mechanism based on preference extravasation of nanoparticles that access the blood-brain barrier (BBB or tumor). Nanoparticles only access the tumor because they have more retention effects than other particles that do not access the tumor.

\section{Peptide vaccine}

In nanoparticle, peptide vaccine helps to target the single or multiple Nano antigens. Some peptide vaccines are EGFRviii, IL13RA2, AIM-2, and MAGE-19 [27,28]. These vaccines are more responsive in patients and tumor patients survived 26 months on average. Peptide vaccine can cause profusion of the cell which does not indicate the targeted antigens. Targeting multiple antigens was the most effective treatment of the tumor [29].

\section{Administration Techniques for Molecular Therapeutics}

Molecular targeting agents can be administrated toward the targeted site by systematic, intracranial and cell-mediated delivery.

\section{Systematic administration}

Systematic Administration of targeted therapeutics is a very easy technique for cargo transportation involving dreary quantity [13]. One evident technique for transportation of these molecules is through Intravenous (IV) injections. The nanoparticle established remedial are frequently administrated in many doses to control the growth of the tumors [30]. Low-frequency enrapt ultrasound supplies confined interference of BBB [31-33]. The preclinical studies have shown that this technique can purely increase the central delivery of the remedial agents into tumor cells [31]. Another method to ease the non -invasion technique is Magnetic targeting at a specialized site $[34,35]$ has been practiced in clinical trials (Clinical Trails Gov. Identifier NCT0005495, NCT00034333). It is reported that magnetic targeting produces a 5times enhancement in the total subjection of glioma cells to the nanoparticle over not specific and non- targeting tumors and increase in target selection for collection in the tumor versus normal brain cells [13]. The main drawbacks of systemic delivery are the danger for the accumulation of nanoparticle in Nano-targeted organs like the liver, kidney, and lungs. The nanoparticle is not hazardous but the longtime interaction of nanoparticle deposition in the brain has not fully addressed [36].

\section{Intracranial administration}

Delivery of therapeutic agent to the targeted site in the brain tumor is a hard job without affecting normal cells. We can enter a therapeutic agent directly into the tumor site that can easily pass the BBB barrier [37]. We can use polymers that are capable to target tumor cells while sparing normal cells. These polymers can be degradable or non-degradable [38]. This method is mostly used when localization of drug to the target is low. It increases the concentration of such an agent within affected cells only. It depends on transporting a large amount of therapeutic agent in a continuous flow [39].

\section{Cell-mediated administration}

Cell-mediated delivery of synthetic nanoparticle-based drug carrier is a very promising strategy to control the distribution of the drug and improve targeting. In this method, cells are found the ability to cross the tumor. Mesenchymal stem cell (MSC) and neural stem cells show the ability to target the brain tumor cells by nanoparticle.

MSCs with bound silica Nano rattle-doxorubicin can be targeted in vivo and in vitro ways on the tumor [40]. It was noticed that Nano capsule containing ferrociphenol can be entered into the tumor and can slow the growth of the tumor [41].

The ability of ph-mediated drug release capabilities. This can deliver doxorubicin conjugated to BBB. This method of combining stem cells and nanoparticle for treatment based upon local administration method.[36]

Another way of treatment is that to combine the carriers and nanoparticles and carriers are tracked via trucker. For this purpose iron oxide was used [40].

\section{Issues and Limitations of Brain-Targeted Therapies}

Development of targeted vehicle is only possible after complete understanding of the target. As the brain is complex in nature so it is very difficult to recognize the specific differences between the target and other cells [6]. Non-covalent drug delivery system often results in the release of drug at a non-specific point during its transport. Some targeted molecules are also degraded by the enzymes. Another problem is safety. Some of the targeted molecules cause toxicity problems. e.g. Nanoparticles show slow toxicity after their entry in the systemic circulation and their mechanism of action are not fully known. So, there is no idea of the appearance of any side effect due to their long-term accumulation on the targeted site. Most of the targeted approaches are used with conventional 
methods of treatment i.e. chemotherapy and radiotherapy for disease management [13]. It limits the use of targeted molecules that target a number of pathways involved in the formation of tumor i.e. broad ranging therapeutic agent must be used [2].

\section{Conclusion}

A number of brain tumor patients are being treated by the targeted therapies which have many advantages over conventional treatment techniques e.g. surgery, radiation, and chemotherapy. The therapeutic agents have many advantages and one of the potential advantages is that these can easily cross the barriers that protect the brain and target only the malignant site and is not harmful to the normal healthy cells of the brain. But it also has some dark aspects i.e. sometimes, it causes non-specific transport, and also sometimes before reaching the site of action these are degraded by the enzymes, and, toxicity profiles. Although these therapeutic agents have some issues and limitations they are mostly used in combination with conventional techniques to improve their efficacy.

\section{References}

1. AM Chacko, C Li, DA Pryma, S Brem, G Coukos, et al. (2013) Targeted delivery of antibody-based therapeutic and imaging agents to CNS tumors: crossing the blood-brain barrier divide. Expert opinion on drug delivery 10: 907-926.

2. AA Nageswara Rao, J Scafidi, EM Wells, RJ Packer (2012) Biologically targeted therapeutics in pediatric brain tumors. Pediatric neurology 46: 203-211.

3. A Toth, S Veszelka, S Nakagawa, M Niwa, MA Deli (2011) Patented in vitro blood-brain barrier models in CNS drug discovery. Recent patents on CNS drug discovery 6: 107-118.

4. KA Witt, TJ Gillespie, JD Huber, RD Egleton, TP Davis (2001) Peptide drug modifications to enhance bioavailability and blood-brain barrier permeability. Peptides 22: 2329-2343.

5. LA Cooper, DA Gutman, Q Long, BA Johnson, SR Cholleti, et al. (2010) The proneural molecular signature is enriched in oligodendrogliomas and predicts improved survival among diffuse gliomas. PloS one 5: e12548.

6. SE El-Habashy, AM Nazief, CE Adkins, MM Wen, AH El-Kamel, et al. (2014) Novel treatment strategies for brain tumors and metastases. Pharmaceutical patent analyst 3: 279-296.

7. K Malpass (2012) Neuro-oncology: Identification of novel glioblastomaassociated antigens reveals targets for immunotherapy, Nature reviews. Neurology 8: 240.

8. DN Louis, H Ohgaki, OD Wiestler, WK Cavenee, PC Burger, et al. (2007) The 2007 WHO classification of tumours of the central nervous system. Acta neuropathologica 114: 97-109.

9. MD Taylor, PA Northcott, A Korshunov, M Remke, YJ Cho, et al. (2012) Molecular subgroups of medulloblastoma: the current consensus. Acta neuropathologica, 123: 465-472.

10. DH Rowitch, AR Kriegstein (2010) Developmental genetics of vertebrate glial-cell specification. Nature 468: 214-222.

11. P Zhang, L Hu, Q Yin, Z Zhang, L Feng, Y Li (2012) Transferrin-conjugated polyphosphoester hybrid micelle loading paclitaxel for brain-targeting delivery: synthesis, preparation and in vivo evaluation. Journal of controlled release: official journal of the Controlled Release Society 159: 429-434.

12. PK Sneed, PH Gutin, DA Larson, MK Malec, TL Phillips, MD Prados, et al. (1994) Patterns of recurrence of glioblastoma multiforme after external irradiation followed by implant boost, International journal of radiation oncology. biology, physics 29: 719-727.
13. Y Cheng, RA Morshed, B Auffinger, AL Tobias, MS Lesniak (2014) Multifunctional nanoparticles for brain tumor imaging and therapy. Advanced drug delivery reviews 66: 42-57.

14. H Wolburg, A Lippoldt (2002) Tight junctions of the blood-brain barrier: development, composition and regulation. Vascular pharmacology 38: 323-337.

15. X Wei, X Chen, M Ying, W Lu (2014) Brain tumor-targeted drug delivery strategies. Acta pharmaceutica Sinica. B 4: 193-201.

16. A Beduneau, P Saulnier, JP Benoit (2007) Active targeting of brain tumors using nanocarriers. Biomaterials 28: 4947-4967.

17. R Spector (2010) Nature and consequences of mammalian brain and CSF efflux transporters: four decades of progress. Journal of neurochemistry 112: 13-23.

18. CE Eyler, WC Foo, KM LaFiura, RE McLendon, AB Hjelmeland, et al. (2008) Brain cancer stem cells display preferential sensitivity to Akt inhibition. Stem cells (Dayton, Ohio) 26: 3027-3036.

19. W Lu, YZ Tan, KL Hu, XG Jiang (2005) Cationic albumin conjugated pegylated nanoparticle with its transcytosis ability and little toxicity against blood-brain barrier. International journal of pharmaceutics 295: 247-260.

20. J Du, WL Lu, X Ying, Y Liu, P Du, et al. (2009) Dual-targeting topotecan liposomes modified with tamoxifen and wheat germ agglutinin significantly improve drug transport across the blood-brain barrier and survival of brain tumor-bearing animals. Molecular pharmaceutics 6: 905-917.

21. B Gupta, TS Levchenko, VP Torchilin (2005) Intracellular delivery of large molecules and small particles by cell-penetrating proteins and peptides. Advanced drug delivery reviews 57(4): 637-651.

22. PR Lockman, DD Allen (2002) The transport of choline, Drug development and industrial pharmacy 28: 749-771.

23. P Ponka, CN Lok (1999) The transferrin receptor: role in health and disease. The international journal of biochemistry \& cell biology 31 : 1111-1137.

24. M Demeule, A Regina, C Che, J Poirier, T Nguyen, et al. (2008) Identification and design of peptides as a new drug delivery system for the brain. The Journal of pharmacology and experimental therapeutics, 324: 1064-1072.

25. NS Ningaraj, M Rao, K Hashizume, K Asotra, KL Black (2002) Regulation of blood-brain tumor barrier permeability by calcium-activated potassium channels. The Journal of pharmacology and experimental therapeutics, 301: 838-851.

26. R Patil, AV Ljubimov, PR Gangalum, H Ding, J Portilla-Arias, et al. (2015) MRI virtual biopsy and treatment of brain metastatic tumors with targeted nanobioconjugates: nanoclinic in the brain. ACS nano 9: 55945608.

27. AB Heimberger, D Suki, D Yang, W Shi, K Aldape (2005) The natural history of EGFR and EGFRvIII in glioblastoma patients. Journal of translational medicine 3: 38.

28. S Phuphanich, CJ Wheeler, JD Rudnick, M Mazer, H Wang, et al. (2013) Phase I trial of a multi-epitope-pulsed dendritic cell vaccine for patients with newly diagnosed glioblastoma. Cancer immunology, immunotherapy: CII 62: 125-135.

29. IF Pollack, RI Jakacki, LH Butterfield, RL Hamilton, A Panigrahy, et al. (2016) Immune responses and outcome after vaccination with gliomaassociated antigen peptides and poly-ICLC in a pilot study for pediatric recurrent low-grade gliomas. Neuro-oncology 18: 1157-1168.

30. Y Cui, Q Xu, PK Chow, D Wang, CH Wang (2013) Transferrin-conjugated magnetic silica PLGA nanoparticles loaded with doxorubicin and paclitaxel for brain glioma treatment. Biomaterials 34: 8511-8520.

31. AB Etame, RJ Diaz, CA Smith, TG Mainprize, K Hynynen, et al. (2012) Focused ultrasound disruption of the blood-brain barrier: a new frontier 
for therapeutic delivery in molecular neurooncology. Neurosurgical focus 32: E3.

32. K Hynynen, N McDannold, N Vykhodtseva, S Raymond, R Weissleder, et al. (2006) Focal disruption of the blood-brain barrier due to $260-\mathrm{kHz}$ ultrasound bursts: a method for molecular imaging and targeted drug delivery. Journal of neurosurgery 105: 445-454.

33. HL Liu, MY Hua, HW Yang, CY Huang, PC Chu, et al. (2010) Magnetic resonance monitoring of focused ultrasound/magnetic nanoparticle targeting delivery of therapeutic agents to the brain. Proceedings of the National Academy of Sciences of the United States of America 107: $15205-15210$.

34. B Shapiro, K Dormer, IB Rutel (2010) A Two-Magnet System to Push Therapeutic Nanoparticles. AIP conference proceedings 1311: 77-88.

35. SD Kong, J Lee, S Ramachandran, BP Eliceiri, VI Shubayev, et al. (2012) Magnetic targeting of nanoparticles across the intact blood-brain barrier. Journal of controlled release: official journal of the Controlled Release Society 164: 49-57.

36. Y Cheng, R Morshed, SH Cheng, A Tobias, B Auffinger, et al. (2013) Nanoparticle-programmed self-destructive neural stem cells for glioblastoma targeting and therapy. Small (Weinheim an der Bergstrasse, Germany) 9: 4123-4129.

37. C Guerin A Olivi, JD Weingart, HC Lawson, H Brem (2004) Recent advances in brain tumor therapy: local intracerebral drug delivery by polymers. Investigational new drugs 22: 27-37.

38. SM Raza, G Pradilla, FG Legnani, QA Thai, A Olivi, et al. (2005) Local delivery of antineoplastic agents by controlled-release polymers for the treatment of malignant brain tumours. Expert opinion on biological therapy 5: 477-494.

39. E Allard, C Passirani, JP Benoit (2009) Convection-enhanced delivery of nanocarriers for the treatment of brain tumors. Biomaterials 30: 23022318.

40. L Li, Y Guan, H Liu, N Hao, T Liu, et al. (2011) Silica nanorattledoxorubicin-anchored mesenchymal stem cells for tumor-tropic therapy. ACS nano 5: 7462-7470.

41. M Roger, A Clavreul, NT Huynh, C Passirani, P Schiller, et al. (2012) Ferrociphenol lipid nanocapsule delivery by mesenchymal stromal cells in brain tumor therapy. International journal of pharmaceutics 423: 6368. 